\title{
The Florajen Digestion Balance Patient Experience Study
}

\author{
Christine Kessler \\ King George, VA, USA \\ Email: ckessler@maranatha.net
}

How to cite this paper: Kessler, C. (2020) The Florajen Digestion Balance Patient Experience Study. Health, 12, 1468-1480. https://doi.org/10.4236/health.2020.1211107

Received: October 14, 2020

Accepted: November 24, 2020

Published: November 27, 2020

Copyright (c) 2020 by author(s) and Scientific Research Publishing Inc. This work is licensed under the Creative Commons Attribution International License (CC BY 4.0).

http://creativecommons.org/licenses/by/4.0/

\begin{abstract}
Background: Antibiotics have long been associated with gastrointestinal (GI) side-effects, leading to antibiotic noncompliance, related morbidities and increased costs. Antibiotic-induced disruption of intestinal microbiota has created interest in prophylactic use of probiotics to prevent antibiotic-associated GI side-effects, with recent trials suggesting GI benefits with concomitant probiotic and antibiotic use. The aim of this study was to see if commercially available Florajen Digestion can help maintain GI health during antibiotic regimens in the community. Methods: This prescriber and pharmacist directed, open label, patient experience study took place between September 2018 and January 2019. Healthcare professionals (HCPs) and patients from 40 states participated. Florajen Digestion probiotic was given to patients free of charge through their prescriber or pharmacist when prescribed or dispensed antibiotics. Surveys were completed by HCPs and patients, who reported on antibiotics prescribed/taken, GI upset, compliance and product satisfaction. Results: A total of $839 \mathrm{HCPs}$ and 404 patients completed the study. Although $63 \%$ of patients reported prior GI side-effects with antibiotic use, only $12 \%$ experienced GI upset with concomitant Florajen Digestion use. Approximately $93 \%$ of patients completed their antibiotic regimen, with 77\% asserting that Florajen Digestion helped them complete their course of antibiotics and $88 \%$ extremely satisfied with the probiotic. Among HCPs, 94\% believed reducing side-effects improved antibiotic compliance, with $88 \%$ stating that probiotics should be recommended concomitantly with oral antibiotics. Conclusion: The results reveal the GI benefits of concomitant use of Florajen Digestion probiotics with antibiotics and support recommendation of Florajen Digestion by HCPs when prescribing or dispensing antibiotics.
\end{abstract}

\section{Keywords}

Probiotics, Antibiotics, Antibiotic-Associated Diarrhea, Clostridium difficile 
Infections, Diarrhea, Gastrointestinal Health, Prevention, Microbiota, Microbiome Dysbiosis

\section{Introduction}

Gastrointestinal (GI) upset is a frequent complication of broad-spectrum antibiotic use that can cause considerable patient distress and reduce treatment compliance. Of particular concern is antibiotic-associated diarrhea (AAD) that occurs in up to $39 \%$ or more of patients receiving antibiotics in the outpatient setting [1]. The defining symptom of AAD is the presence of 3 or more loose stools per day that develops within hours to 8 weeks following antibiotic exposure, with a median onset of 5 to 9 days [2] [3] [4] [5]. The presentation of AAD may be mild and self-limiting diarrhea to severe Clostridium difficile-associated diarrhea and pseudomembranous colitis [5] [6] [7]. Beside impacting patient adherence to antibiotic therapy, antibiotic-related GI side effects can greatly impact morbidity and increase health care costs [8].

The life-saving and transformative impact of antibiotics on medicine cannot be overstated, but their use carries potential risks. Of note is the deleterious impact of antibiotics on intestinal microbiota, a complex community of as many as 100 trillion diverse and interactive microbes that play a fundamental role in health and disease via its influence on metabolism, nutrition, and immune function [2] [9].

It is thought that antibiotics alter GI digestive functions in several ways. Direct toxic effects on the intestinal epithelial lining can produce irritation, increased inflammation, and disruption of nutrient absorption and motility patterns. Antibiotics also disturb the balance of intestinal microbiota leading to reduced abundance and diversity of gut bacteria. These changes impair the microbiota's capacity to resist invasion of pathogenic microorganisms [10] [11] [12] or prevent an overgrowth of endogenous, pathogenic bacteria, some of which may be drug resistant organisms [9]-[14]. Despite the eventual recovery of microbiota counts following antibiotic discontinuation, there can be long-lasting effects on the gut microbiome and patient disease susceptibility [2] [9] [14] [15] [16] [17].

Any type of antibiotic can cause GI distress and AAD. However, clindamycin, fluoroquinolones, and broad-spectrum penicillin and cephalosporins have been found to pose the greatest risk [16]. While it is known that a specific antibiotic can increase a patient's propensity to develop GI complications, so too is the patient's susceptibility. This susceptibility is increased by extremes in age, underlying co-morbidities, immunologic status, and recent hospitalization. Prolonged antibiotic use, oral administration, or taking more than one antibiotic creates further risk of AAD [16] [17] [18] [19].

The best way to manage AAD is to prevent it. To do so, the gut microbiota makes a useful target. In recent years, probiotics have been increasingly utilized as preventive treatment for antibiotic-induced microbiota dysbiosis and asso- 
ciated adverse GI effects. The International Scientific Association for Probiotics and Prebiotics (ISAPP) defines "probiotics" as "supplemental live microorganisms" that, when administered in adequate amounts, may confer multiple health benefits based on data from numerous human studies and positive meta-analyses [20] [21] [22] [23] [24]. The ISAPP consensus panel has further identified ways probiotics may exert their activity on the human microbiome that likely varies depending on microbiota strains or species [21].

Many clinical trials have looked at the effectiveness of probiotics in preventing antibiotic-associated GI complications. Systemic review and meta-analyses suggest that the use of several species and strains of probiotics can have an overall positive effect in preventing $\mathrm{AAD}$, by as much as $51 \%$ in some populations [6] [24] [25] [26] [27]. No study, however, has looked at overall patient satisfaction and compliance to antibiotic therapy when a probiotic is taken to reduce mild to moderate GI effects. The greatest benefit for AAD reduction has been found in patients under the age of 65 years and appears dependent upon probiotic strain, dose and duration [28]. At this time, microbial strains believed to be most effective in treating antibiotic-related GI side-effects include Lactobacillus GG, Lactobacillus acidophilus La-5, Bifidobacterium lactis B-12, and Saccharomyces boulardii (a nonpathogenic yeast) [29] [30] [31] [32].

The 2019 Cochrane report on prevention of AAD recommends the use of high dose probiotics ( $\geq 5$ billion CFUs per day) [23] [27]. Concerns have been raised about the safety of widespread probiotic use. However, a 2014 Agency for Healthcare Research and Quality (AHRQ) assessment concluded that probiotic supplements were generally safe in both young and old for short term use, but that there was insufficient data on long-term safety of these products [24] [33] [34] [35].

The 2012 National Health Interview Survey (NHIS) identified probiotics as the third most commonly used dietary supplement in U.S. adults. Probiotic use quadrupled between 2007 and 2012 with approximately 4 million (1.6 percent) of U.S. adults now using them: a number expected to rise. [36] Because probiotics are "over-the-counter," patients often self-prescribe, potentially making ill-informed choices. Pharmacists and medical providers are in a unique position to help patients navigate the available products and inform them of their benefits, especially with regard to their use with antibiotic therapy. In a recent international survey, $79 \%$ of health care professionals (medical doctors, pharmacists, dentists and allied health professionals) reported advising their patients on probiotic use but nearly $58 \%$ felt the need for more information [37].

Currently, there are no firm, medical guidelines on how probiotics should be administered. Commercial literature on various probiotic supplements offer conflicting instructions on how to take probiotics in relation to meals. Tomkins et al examined the effects of food exposure on probiotic viability during their transit through the GI tract and found dosing 30 minutes before or during food consumption ensured greater probiotic microbial survival [38]. With regard to probiotic use with antibiotics, there remains lack of clarity. A general recom- 
mendation is to take the probiotic within 2 days of starting antibiotic therapy and to continue the probiotic for a minimum of $2-3$ weeks following antibiotic discontinuation. Because living microorganisms comprise these supplements, refrigerated probiotics are recommended to reduce or delay the death of these microbes [39] [40].

\section{Objectives}

This was a prescriber and pharmacist directed, open label patient experience study. The primary objective of this study was to determine whether Florajen Digestion, a commercial probiotic with 3 bacterial strains, could help maintain GI health during antibiotic regimens in a community setting. Other trial objectives were:

1) To understand patient experience and satisfaction with Florajen Digestion.

2) To understand why and which antibiotics would trigger health professionals to recommend Florajen Digestion.

3) To understand healthcare professional (HCP) and patient expectations of probiotic use.

\section{Methods}

The BALANCE study was conducted between September of 2018 and January 2019. Healthcare professionals (HCPs) and patients from 40 states participated in the study. Prescribers (physicians, nurse practitioners, physician assistants) known to write large numbers of antibiotic prescriptions were invited to participate in the study. Pharmacists were recruited from a list of independent pharmacies that purchase, or have purchased, Florajen in the past. Patients were provided a 30-count bottle of refrigerated Florajen Digestion probiotic, free of charge, through their prescriber or pharmacist when recommending or dispensing antibiotics. Florajen Digestion is a commercial probiotic supplement containing a total 15 billion live cultures of Lactobacillus acidophilus La-14, Lactobacillus acidophilus NCFM, Bifidobacterium lactis Bi-07, Bifidobacterium lactis HN019, and Bifidobacterium longum BL-05. Surveys were completed by HCPs and patients, who reported on antibiotics prescribed and taken, compliance, past and/or current antibiotic-related GI upset, and product satisfaction.

Surveys given to HCPs sought their reasons for providing Florajen Digestion to the patient. Among reasons listed from which to choose included belief in the product's efficacy, stability and culture potency, patient satisfaction, antibiotic compliance, and familiarity with the Florajen brand. Further information was elicited about the provider's overall belief in probiotic GI benefits, patients' past history of GI distress while taking antibiotics and which antibiotics would motivate a medical recommendation for probiotics.

Inclusion criteria required that patients were being treated with a first course of antibiotics with a regimen less than or equal to 10 days. Additionally, patients had to be appropriate for probiotic therapy, and not immunocompromised. Pa- 
tient respondents reported on demographic data such as age, sex and ethnicity as well as past history of antibiotic-induced GI side effects.

Patients were instructed to begin taking Florajen Digestion at the start of their antibiotic regimen. The probiotic was to be taken daily 1 to 2 hours before or after their antibiotic and continued at least 2 - 3 weeks after antibiotic discontinuation or until all of the dispensed probiotic was gone. Patients were then asked to complete a survey seeking information about their history of antibiotic use and feedback about general GI health, new GI distress, compliance with the antibiotic and probiotic regimen, and overall satisfaction with Florajen Digestion. Every patient who returned the survey received a $\$ 5$ Starbucks gift card. Healthcare professionals received no compensation.

\section{Results}

1) Prescriber and Pharmacist responses

A total of 831 surveys were completed by HCPs from 40 states. Pharmacists (\#562) surpassed prescribers (\#269) in responses, however, both prescribers and pharmacists were nearly equal in their prioritization of antibiotics that would induce them to recommend Florajen Digestion. Specifically, Penicillins (Amoxicillin, Augmentin, etc), clindamycin, ciprofloxacin and doxycycline ranked as the top 4 antibiotics of concern [Figure 1].

Perceived clinical efficacy was the leading reason for HCPs to recommend Florajen Digestion to patients, although pharmacists placed slightly more importance on compliance, patient satisfaction and stability compared to prescribers.

Over 90 percent of HCPs believed that antibiotic compliance increased when related side effects were reduced [Figure 2]. To that end, most of the participating HCPs felt strongly that probiotics should be recommended when antibiotics

\section{Drug classes selected by over $50 \%$ of Healthcare Professionals $(n=767)$}

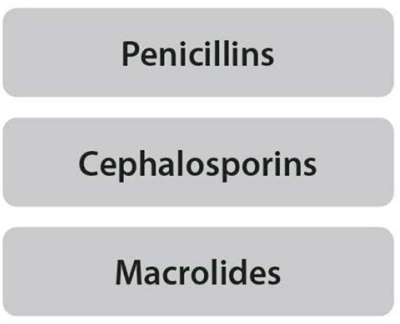

\section{Lincosamides (clindamycin)}

Fluoroquinolones

Tetracyclines

\section{Sulfonamides}

Figure 1. Antibiotic drug classes likely to generate a Florajen digestion recommendation by HCPs. 
Does Antibiotic Compliance Increase with Reduced GI Side Effects?

$(n=813, \mathrm{HCP})$
Should Probiotics Be Recommended Concomitantly with Oral Anitbiotics to Reduce the Risk of GI Side-Effects? $(n=789, H C P S)$

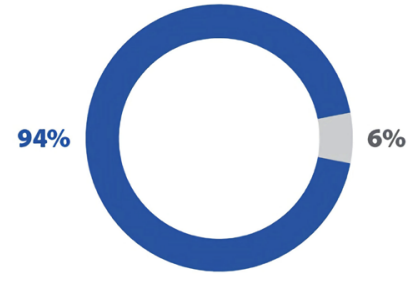

Yes No

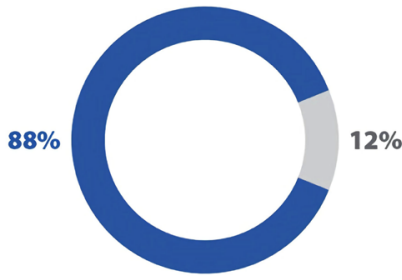

Yes No

Figure 2. HCPs believe that antibiotic compliance increases with reduced GI side-effects and that Probiotics given concomitantly can reduce GI side-effects.

were prescribed to prevent or minimize GI side effects. A small percentage $(<15 \%)$ of HCPs reported that they would only recommend probiotics if patients expressed a desire for them [Figure 2]. Despite HCP-stated acceptance of probiotic GI benefits when taking oral antibiotics, nearly two-thirds of HCPs testified that they did not know if the patient had previously experienced prior antibiotic-related side effects [Figure 3].

2) Patient Responses and Demographics

A total of 404 surveys were completed by patients who received Florajen Digestion from an HCP. The majority of patient respondents were Caucasian, female and represented a wide range in age [Table 1]. Interestingly, nearly half of pharmacist-initiated patient surveys came from patients over 60 years of age, whereas those under 60 years comprised nearly $70 \%$ of prescriber-initiated patient surveys.

A surprising majority of patients (59\% to $69 \%$ ) reported a past history of antibiotic-related GI side-effects, a higher incidence than reported in the literature [Table 1]. In this study, only $12 \%$ of patients reported GI side effects, which occurred in patients with and without a history of antibiotic GI upset [Figure 4]. No single antibiotic was identified as a primary perpetrator of GI upset.

Most compelling was that an average of $93 \%$ of patients completed their antibiotic regimen, with 77\% claiming Florajen Digestion had helped them do so [Figure 4].

Forty one percent (41\%) of patient respondents in this study revealed they had tried other probiotics with past antibiotic therapy; Culturelle and acidophilus were the most commonly cited. None reported prior Florajen Digestion use, however $76 \%$ of patients who tried other probiotics for antibiotic-associated GI side-effects, preferred Florajen Digestion [Figure 5]. It is not known if their probiotic choices were HCP-recommended.

Although a wide variety of antibiotics were prescribed to participating patients, Amoxicillin and Augment topped the list with nearly 30\% of patient respondents 
Table 1. Patient demographics.

\begin{tabular}{|c|c|c|c|c|c|c|c|}
\hline \multicolumn{8}{|c|}{ Patient Demographics and Clinical History } \\
\hline \multicolumn{2}{|l|}{$\begin{array}{c}\text { Ethnicity } \\
\mathrm{n}=373\end{array}$} & \multicolumn{2}{|l|}{$\begin{array}{c}\text { Age } \\
\mathrm{n}=354\end{array}$} & \multicolumn{2}{|l|}{$\begin{array}{l}\text { Gender } \\
\mathrm{n}=393\end{array}$} & \multicolumn{2}{|c|}{$\begin{array}{l}\text { Percentage of patients reporting a history of } \\
\text { antibiotic -associated GI side-effects } \\
\qquad n=384\end{array}$} \\
\hline Caucasian & $74 \%$ & $<30$ & $20 \%$ & Female & $70 \%$ & Yes & $63 \%$ \\
\hline African-American/Black & $7 \%$ & $30-45$ & $18 \%$ & Male & $30 \%$ & No & $28 \%$ \\
\hline Hispanic & $5 \%$ & $46-60$ & $22 \%$ & & & Don’t Know & $9 \%$ \\
\hline Asian & $10 \%$ & $61-75$ & $23 \%$ & & & & \\
\hline Middle Eastern & $2 \%$ & $75+$ & $17 \%$ & & & & \\
\hline Other & $2 \%$ & & & & & & \\
\hline
\end{tabular}

\section{Did Your Patient Have a Prior History of Antibiotic-} Associated Side Effects? ( $n=814$, HCPs)

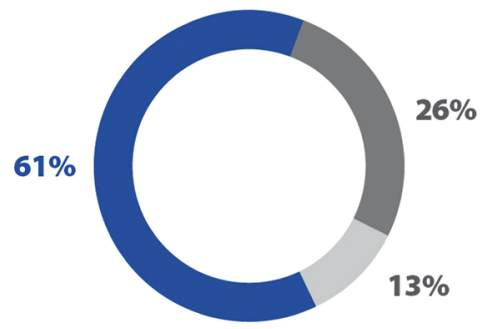

$$
\begin{array}{lll}
\text { Unknown } & \begin{array}{l}
\text { Has a history } \\
\text { of antibiotic } \\
\text { side-effects }
\end{array} & \begin{array}{l}
\text { Does not has a } \\
\text { history of antibiotic } \\
\text { side-effects }
\end{array}
\end{array}
$$

Figure 3. Percent of HCPs reporting they did not know if patients had prior history of antibiotic-associated side-effects.

\section{Did Your Patient Experience GI Side-Effects During this \\ Study? \\ ( $n=384, \mathrm{HCPS})$}

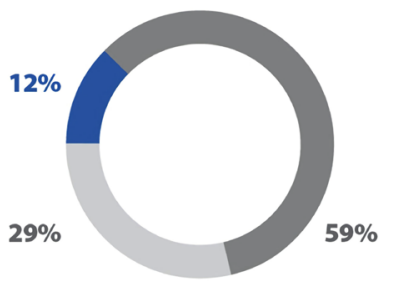

Patients that experienced

Gl side-effects were on a

wide variety of antibiotics:

no one antibiotic was

the primary cause of

side-effects

\section{Did Florajen Digestion Help Patients Finish Their Course of Anitbiotics?}

$(n=389, H C P$ s)

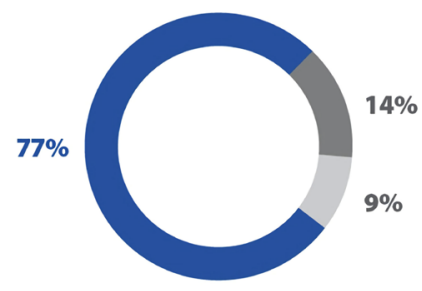

$\square$ Yes No Don't Know

Figure 4. Percent of patients experiencing GI side-effects during this study and HCP perspective on whether Florajen digestion helped patients finish their course of antibiotics. 


\section{Patients Who Have Tried Other Probiotics for Antibiotic- Associated GI Side-Effects AND Prefer Florajen Digestion} ( $n=109$, patients)

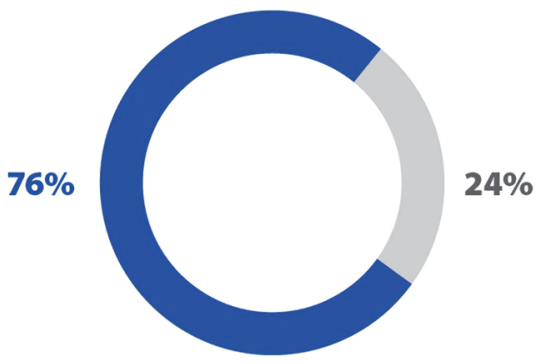

Yes No

Figure 5. Percent of patients who have tried other probiotics and prefer Florajen digestion.

reporting that they were prescribed these two drugs. The duration of antibiotic and probiotic use ranged from 3 to 10 days with an average of 7 treatment days [Figure 6].

Overall satisfaction with Florajen Digestion was high at 88\% [Figure 7].

\section{Discussion}

Gastrointestinal distress, including AAD, has emerged as a common complication of antibiotic use which can limit compliance to treatment regimen. Adverse GI side effects are believed to be related to antibiotic-induced disruption of existing intestinal microbiota and changes to intestinal epithelial integrity. Many outpatient and inpatient clinical trials have assessed prophylactic probiotic use with antibiotics to prevent, or minimize, GI side effects. The evidence suggests a moderate protective effect of probiotics in preventing AAD. Despite these findings, there remains a hesitancy among community-based HCPs to recommend probiotic use with antibiotics. This is not surprising, as many clinical trials have been small with researchers using varied, proprietary bacterial strains in their probiotic cocktails. Should HCPs choose to recommend the use of probiotics, they are only available to the public as over-the-counter supplements. To date, there is insufficient research to determine the efficacy of these commercial probiotic supplements in preventing antibiotic-related GI distress.

From the results of this study, it appears that a large majority of HCPs (94\%) believe that reducing GI side-effects can increase antibiotic compliance, with most supporting prophylactic probiotic use with oral antibiotics. Despite agreeing that GI distress can negatively impact antibiotic compliance, a majority of 


\section{Patients Submitting Feedback had been Prescribed a Wide Range of Antibiotics for an Average of 7.2 Days Antibiotics prescribed (patient reported) \\ ( $n=390$, patients)}

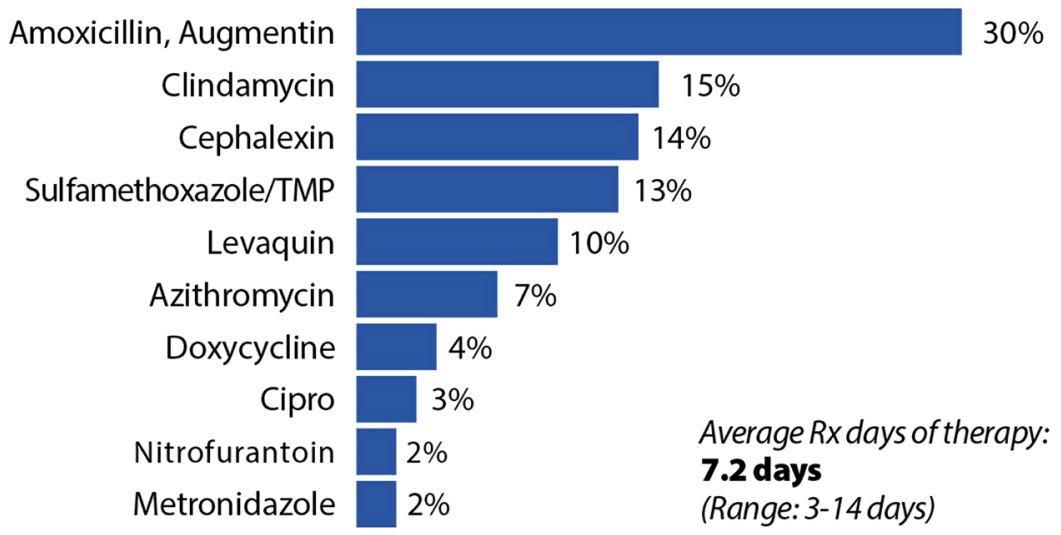

Figure 6. Antibiotics prescribed to patient respondents (patient reported).

\section{Patient Satisfaction with Florajen \\ ( $n=383$, patients)}

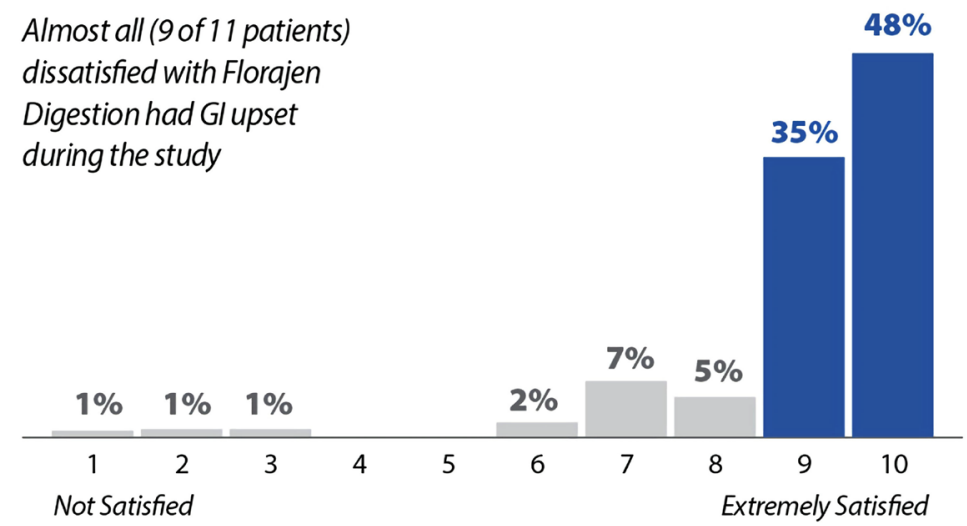

Figure 7. Percent of patients by level of satisfaction with Florajen digestion.

HCPs did not know if their patient had a history of antibiotic complications prior to prescribing the current antibiotic. This finding is concerning as it limits early identification of patients with higher risk for AAD and other GI upset.

While the literature often cites the incidence of antibiotic-related GI side-effects as high as $49 \%$, the reported incidence of past antibiotic-associated GI distress in this study was considerably higher (63\%) possibly indicating underreporting of these adverse effects or the increased participation of patients who had expe- 
rienced antibiotic associated GI upset in the past. Despite the high prior occurrence of GI sequela, only $12 \%$ of participating patients in the BALANCE study reported GI side-effects. This may explain the very high compliance to both antibiotic and probiotic therapy ( $>91 \%)$ in this study, with most patients claiming Florajen Digestion helped them finish their course of antibiotics. Patients who did not fully comply with, or complete, their antibiotic treatment plan, identified GI-upset as the cause.

Perhaps mirroring the public at large, a majority of BALANCE study patients reported past use of probiotics for antibiotic-associated GI side-effects, although none testified to prior use of a Florajen product. A large majority of the patients surveyed, including those with a history of past probiotic use with antibiotics, reported "extreme satisfaction" with Florajen Digestion and 89\% declared their intent to use this probiotic product with antibiotics in the future.

This was the first open label, patient experience study examining the concomitant use of a commercial probiotic supplement with antibiotic therapy in an outpatient setting. The strength of this study was the large number of HCPs and patients who chose to participate within the short trial time frame, providing good insight into HCP probiotic prescribing patterns as well as overall compliance with antibiotic and probiotic regimens. However, the study was limited by the subjective nature of the patients' responses as well as lack of ascertaining the exact nature of antibiotic-associated GI side-effects experienced by patients.

\section{Conclusion}

The Florajen Digestion BALANCE study provided feedback from hundreds of health care prescribers, pharmacists and patients to help gain insight about the GI health benefits of concomitant use of Florajen Digestion, a commercial probiotic supplement, with antibiotics. Results from this study support the recommendation of Florajen Digestion by HCPs when prescribing or dispensing antibiotics to help prevent GI side effects.

\section{Funding}

Clarion Brands supplied Floraen Digestion supplements for this study as well as funding for the authorship of this publication.

\section{Conflicts of Interest}

The author declared no potential conflicts of interest with respect to the research, authorship, and/or publication of this article.

\section{References}

[1] McFarland, L.V. (1998) Epidemiology, Risk Factors and Treatments for Antibiotic-Associated Diarrhea. Digestive Diseases, 16, 292-307.

https://doi.org/10.1159/000016879

[2] Bartlett, J.G. (2002) Antibiotic Associated Diarrhea. The New England Journal of 
Medicine, 346, 334-339. https://doi.org/10.1056/NEJMcp011603

[3] Allen, S.J., Wareham, K., Wang, D., et al. (2013) Lactobacilli and bifidobacteria in the Prevention of Antibiotic-Associated Diarrhoea and Clostridium difficile Diarrhoea in Older Inpatients (PLACIDE): A Randomised, Double-Blind, Placebo-Controlled, Multicentre Trial. Lancet, 382, 1249-1257. https://doi.org/10.1016/S0140-6736(13)61218-0

[4] Butler, C., Duncan, D. and Hood, K. (2012) Does Taking Probiotics Routinely with Antibiotics Prevent Antibiotic Associated Diarrhoea? BMJ, 344, e682. https://doi.org/10.1136/bmj.e682

[5] McFarland, L.V. (2008) Antibiotic-Associated Diarrhea: Epidemiology, Trends and Treatment. Future Microbiology, 3, 563-578. https://doi.org/10.2217/17460913.3.5.563

[6] Blaabjerg, S., Artzi, D.M. and Aabenhus, R. (2017) Probiotics for the Prevention of Antibiotic-Associated Diarrhea in Outpatients-A Systematic Review and Meta-Analysis. Antibiotics, 6, 21-37. https://doi.org/10.3390/antibiotics6040021

[7] Giannelli, F.R. (2017) Antibiotic-Associated Diarrhea. Journal of the American Academy of Physician Assistants, 30, 46-47. https://doi.org/10.1097/01.JAA.0000524721.01579.c9

[8] Turck, D., Bernet, J.-P., Marx, J., Kempf, H., Giard, P., Walbaum, O., et al. (2003) Incidence and Risk Factors of Oral Antibiotic-Associated Diarrhea in an Outpatient Pediatric Population. Journal of Pediatric Gastroenterology and Nutrition, 37, 22-26. https://doi.org/10.1097/00005176-200307000-00004

[9] Yoon, Y.I. and Yoon, S.Y. (2018) Disruption of the Gut Ecosystem by Antibiotics. Yonsei Medical Journal, 59, 4-12. https://doi.org/10.3349/ymj.2018.59.1.4

[10] Keeney, K.M., Yurist-Doutsch, S., Arrieta, M.-C. and Finlay, B.B. (2014) Effects of Antibiotics on Human Microbiota and Subsequent Disease. Annual Review of Microbiology, 68, 217-235. https://doi.org/10.1146/annurev-micro-091313-103456

[11] Beaugerie, L. and Petit, J.-C. (2004) Microbial-Gut Interactions in Health and Disease: Antibiotic-Associated Diarrhoea. Best Practice \& Research Clinical Gastroenterology, 18, 337-352. https://doi.org/10.1016/j.bpg.2003.10.002

[12] Servin, A.L. (2004) Antagonistic Activities of Lactobacilli and Bifidobacteria against Microbial Pathogens. FEMS Microbiology Reviews, 28, 405-440.

https://doi.org/10.1016/j.femsre.2004.01.003

[13] Francino, M.P. (2016) Antibiotics and the Human Gut Microbiome: Dysbioses and Accumulation of Resistances. Frontiers in Microbiology, 6, 1-11. https://doi.org/10.3389/fmicb.2015.01543

[14] Modi, S.R., Collins, J.J. and Relman, D.A. (2014) Antibiotics and the Gut Microbiota. Journal of Clinical Investigation, 124, 4212-4218. https://doi.org/10.1172/JCI72333

[15] Croswell, A., Amir, E., Teggatz, P., Barman, M. and Salzman, N.H. (2009) Prolonged Impact of Antibiotics on Intestinal Microbial Ecology and Susceptibility to Enteric Salmonella Infection. Infection and Immunity, 77, 2741-2753. https://doi.org/10.1128/IAI.00006-09

[16] Dethlefsen, L. and Relman, D.A. (2011) Incomplete Recovery and Individualized Responses of the Human Distal Gut Microbiota to Repeated Antibiotic Perturbation. Proceedings of the National Academy of Sciences of the United States of America, 108, 4554-4561. https://doi.org/10.1073/pnas.1000087107

[17] Song, H.J., Kim, J.-Y., Jung, S.-A., et al. (2010) Effect of Probiotic Lactobacillus (La- 
cidofil $^{\Phi}$ Cap) for the Prevention of Antibiotic-Associated Diarrhea: A Prospective, Randomized, Double-Blind, Multicenter Study. Journal of Korean Medical Science, 25, 1784-1791. https://doi.org/10.3346/jkms.2010.25.12.1784

[18] Hull, M.W. and Beck, P.L. (2004) Clostridium Difficile-Associated Colitis. Canadian Family Physician, 50, 1536-1545.

[19] Wilcox, M.H. (2003) Gastrointestinal Disorders and the Critically Ill: Clostridium Difficile Infection and Pseudomembranous Colitis. Best Practice \& Research Clinical Gastroenterology, 17, 475-493. https://doi.org/10.1016/S1521-6918(03)00017-9

[20] Lönnermark, E., Friman, V., Lappas, G., et al. (2010) Intake of Lactobacillus plantarum Reduces Certain Gastrointestinal Symptoms during Treatment with Antibiotics. Journal of Clinical Gastroenterology, 44, 106-112. https://doi.org/10.1097/MCG.0b013e3181b2683f

[21] Gibson, G.R., Hutkins, R., Sanders, M.E., Prescott, S.L., Reimer, R.A., Salminen, S.J., et al. (2017) Expert Consensus Document: The International Scientific Association for Probiotics and Prebiotics (ISAPP) Consensus Statement on the Definition and Scope of Prebiotics. Nature Reviews Gastroenterology \& Hepatology, 14, 491-502. https://doi.org/10.1038/nrgastro.2017.75

[22] Hill, C., Guarner, F., Reid, G., Gibson, G.R., Merenstein, D.J., Pot, B., et al. (2014) Expert Consensus Document: The International Scientific Association for Probiotics and Prebiotics Consensus Statement on the Scope and Appropriate Use of the Term Probiotic. Nature Reviews Gastroenterology \& Hepatology, 11, 506-514. https://doi.org/10.1038/nrgastro.2014.66

[23] Guo, Q., Goldenberg, J.Z., Humphrey, C., El Dib, R. and Johnston, B.C. (2019) Probiotics for the Prevention of Pediatric Antibiotic-Associated Diarrhea. Cochrane Database of Systematic Reviews, 4, CD004827. https://doi.org/10.1002/14651858.CD004827.pub5

[24] Hempel, S., Newberry, S.J., Maher, A.R., Wang, Z., Miles, J.N., et al. (2012) Probiotics for the Prevention and Treatment of Antibiotic-Associated Diarrhea: A Systematic Review and Meta-Analysis. JAMA, 307, 1959-1969. https://doi.org/10.1001/jama.2012.3507

[25] Ashraf, R. and Shah, N.P. (2014) Immune System Stimulation by Probiotic Microorganisms. Critical Reviews in Food Science and Nutrition, 54, 938-956. https://doi.org/10.1080/10408398.2011.619671

[26] Lau, C.S. and Chamberlain, R.S. (2016) Probiotics Are Effective at Preventing Clostridium Difficile-Associated Diarrhea: A Systematic Review and Meta-Analysis. International Journal of General Medicine, 9, 27-37. https://doi.org/10.2147/IJGM.S98280

[27] Goldenberg, J., Lytvyn, L., Steurich, J., Parkin, P., Mahant, S. and Johnston, B. (2015) Probiotics for the Prevention of Pediatric Antibiotic-Associated Diarrhea. Cochrane Database of Systematic Reviews, 12, CD004827. https://doi.org/10.1002/14651858.CD004827.pub4

[28] Jafarnejad, S., Shab-Bidar, S., Speakman, J.R., Parastui, K., Daneshi-Maskooni, M. and Djafarian, K. (2016) Probiotics Reduce the Risk of Antibiotic-Associated Diarrhea in Adults (18-64 Years) but Not the Elderly (>65 Years): A Meta-Analysis. Nutrition in Clinical Practice, 31, 502-513. https://doi.org/10.1177/0884533616639399

[29] Szajewska, H. and Kołodziej, M. (2015) Systematic Review with Meta-Analysis: Lactobacillus rhamnosus GG in the Prevention of Antibiotic-Associated Diarrhoea in Children and Adults. Alimentary Pharmacology \& Therapeutics, 42, 1149-1157. 
https://doi.org/10.1111/apt.13404

[30] Szajewska, H. and Kołodziej, M. (2015) Systematic Review with Meta-Analysis: Saccharomyces boulardii in the Prevention of Antibiotic-Associated Diarrhoea. Alimentary Pharmacology \& Therapeutics, 42, 793-801. https://doi.org/10.1111/apt.13344

[31] Schnadower, D., Tarr, P.I., Casper, T.C., Gorelick, M.H., Dean, J.M., O’Connell, K.J., et al. (2018) Lactobacillus rhamnosus GG versus Placebo for Acute Gastroenteritis in Children. The New England Journal of Medicine, 379, 2002-2014. https://doi.org/10.1056/NEJMoa1802598

[32] Sniffen, J.C., McFarland, L.V., Evans, C.T. and Goldstein, E.J.C. (2018) Choosing an Appropriate Probiotic Product for Your Patient: An Evidence-Based Practical Guide. PLoS ONE, 13, e0209205. https://doi.org/10.1371/journal.pone.0209205

[33] Agamennone, V., Krul, C.A.M., Rijkers, G. and Kort, R. (2018) A Practical Guide for Probiotics Applied to the Case of Antibiotic-Associated Diarrhea in The Netherlands. BMC Gastroenterology, 18, Article No.: 103. https://doi.org/10.1186/s12876-018-0831-x

[34] Stadlbauer, V. (2015) Immunosuppression and Probiotics: Are They Effective and Safe? Beneficial Microbes, 6, 823-828. https://doi.org/10.3920/BM2015.0065

[35] Didari, T., Solki, S., Mozaffari, S., Nikfar, S. and Abdollahi, M. (2014) A Systematic Review of the Safety of Probiotics. Expert Opinion on Drug Safety, 13, 227-239. https://doi.org/10.1517/14740338.2014.872627

[36] https://nccih.nih.gov/research/statistics/NHIS/2012/natural-products/biotics

[37] Fijan, S., Frauwallner, A., Varga, L., Langerholc, T., Rogelj, I., Lorber, M., Lewis, P. and Bržan, P.P. (2019) Health Professionals' Knowledge of Probiotics: An International Survey. International Journal of Environmental Research and Public Health, 16, 3128. https://doi.org/10.3390/ijerph16173128

[38] Tompkins, T.A., Mainville, I. and Arcand, Y. (2011) The Impact of Meals on a Probiotic during Transit through a Model of the Human Upper Gastrointestinal Tract. Beneficial Microbes, 2, 295-303. https://doi.org/10.3920/BM2011.0022

[39] Hogan, D.E., Ivanina, E.A. and Robbins, D.H. (2018) Probiotics: A Review for Clinical Use. Gastroenterology \& Endoscopy News, October 2018, 1-7.

[40] Ritchie, M.L. and Romanuk, T.N. (2012) A Meta-Analysis of Probiotic Efficacy for Gastrointestinal Diseases. PLoS ONE, 7, e34938. https://doi.org/10.1371/journal.pone.0034938 